reference text in this country for a substantial period of time in the future.

This relatively unique position rests not only on the fact that it is a clear and accurate version of quantum mechanics, for other books such as those of Rojansky and Kemble have the same virtues. This book has the additional virtue that it is intended to prepare the reader for an understanding of the frontier problems of quantum mechanics as they are being attacked at the present time by theoretical physicists. That is, the book has what might be termed vector content in the sense that the student not only obtains an appreciation of the problems in which non-relativistic quantum mechanics has triumphed in treating atomic and nuclear problems, but is also guided systematically to the frontier problems of relativistic field theories which play so important a role in current developments of the physics of high energy particles. Perhaps the last introductory account of the subject which attempted to do this is Pauli's Die allgemeine Prinzipien der Wellenmechanik which appeared in the Handbuch der Physik, vol. 24, in 1933.

The book contains 14 chapters and may be divided into three parts. In Schiff's own words, "The first three chapters constitute an introduction to quantum mechanics, in which the physical concepts are discussed and the Schrödinger wave formalism is established. The next eight chapters comprise the central part of the book. This part presents exact solutions of the wave equation for both energy-level and collision problems, the Heisenberg matrix formalism and transformation theory, approximation methods, radiation theory, and some applications to atomic systems. Since the first eleven chapters correspond to a typical one-year graduate course, it seemed desirable to include a semi-classical treatment of electromagnetic radiation in the central part of the book (Chapter X) even though some of the results are obtained again in Chapter XIV. The last part of the book corresponds to a short course in what is often called advanced quantum mechanics. It consists of relativistic particle theory and an introduction to quantized field theory and quantum electrodynamics."

\title{
Frederick SeItz
}

Plastic deformation. By L. N. Kachanov, N. M. Beliaev, A. A. Ilyushin, W. Mostow, and A. N. Gleyzal. Ed. by H. H. Hausner. New York, Mapleton, 1948. 192 pp. \$8.00.

This book consists of a set of seven independent papers on plasticity. Five of these appeared originally in Russian periodicals and the 
two remaining ones are based on technical reports prepared under the auspices of the U. S. Navy. Advance notices of this book gave the impression that it forms a coherent account of the current state of art in the field of plasticity. This is far from being the case. The rationale followed in the selection of papers is not clear and the choice appears to be almost random.

The book is divided into seven chapters corresponding to seven distinct papers reproduced in it. The first five of these are photo-offset reproductions of mimeographed free translations of original articles prepared by the Applied Mathematics Group of Brown University for the use of the personnel of the David Taylor Model Basin. They appear as:

Chapter 1, On the mechanics of plastic solids, a 5-page largely synoptic article originally published in Prikladnaia Matematika i Mekhanika vol. 4 (1940) pp. 37-42, by L. N. Kachanov.

Chapter 2, Theories of plastic deformation, Izvestia, Acad. Sci. USSR, Technical Series (1937) pp. 49-70, by N. M. Beliaev.

Chapters 3, 4, and 5 consist of three significant papers by A. A. Ilyushin, (a) Some problems in the theory of plastic deformations, Prikladnaia Matematika i Mekhanika vol. 7 (1943) pp. 245-272. (b) Relation between the theory of Saint Venant-Levy-Mises and the theory of small elastic-plastic deformations, ibid. vol. 9 (1945) pp. 207218. (c) The theory of small elastic-plastic deformations, ibid. vol. 10 (1946) pp. 347-356.

The two remaining chapters are drawn from a report entitled Plastic deformations of thin plates under hydrostatic pressure, prepared by W. Mostow for the U. S. Navy Bureau of Ships, and a report by A. A. Gleyzal on Plastic deformation of a thin circular plate under pressure, which originally appeared as David Taylor Model Basin Report No. 532 (1946).

The reproduction is below average in quality. Some mimeographed material did not photograph well and the publishers had it retyped and introduced so many errors in the process as to make several pages unintelligible. An indication of the careless way in which the book was thrown together may be gleaned from the short page of introduction which contains no less than a dozen errors including the misspellings of the names of three authors of this book. For some reason the backbone and cover carry only the name of L. N. Kachanov whose contribution to the volume consists of a paper occupying less than five typewritten pages.

The usefulness of this book lies only in the fact that it makes translations of several Russian papers readily available. 
The reviewer feels impelled to call attention to the questionable ethical conduct of the publishers. Despite the fact that all papers reproduced in this volume are in the public domain, the book bears the notice of copyright by the Mapleton House. It is certain that no authors' or translators' permission was obtained by the publisher to reproduce this material. Considering the inexpensive photo-offset mode of reproduction of existing material, the price of the book is exorbitant.

\section{S. SoKolnIKoFF}

Mathematical biophysics. By Nicolas Rashevsky. Rev. ed. University of Chicago Press, 1948. 23+669 pp. $\$ 7.50$.

The wide attention and considerable acclaim given to the first publication of Rashevsky's Mathematical biophysics in 1938 was due in large part to the rich promise contained in that book. Those mathematicians who loved to see mathematics in the role of fathoming nature's secrets viewed mathematical biophysics as a new area of conquest. Those biologists who were "monists" or "reductionists" at heart hailed it as the much needed link between their own methods and those of mathematical physics.

The revised edition gives ample evidence that the promise is being fulfilled. Almost twice as large as the original publication, the revised edition includes in its pages ten years' progress in mathematical biology.

The progress is largely due to the work of Rashevsky himself and that of his principal collaborators, who are or have been members of the Committee on Mathematical Biology at The University of Chicago. The work covers a wide range of topics, but essentially it can be viewed as pursuing two main directions: (1) The extension of the theory and methodology; (2) The applications of the theory.

With regard to the first direction, an important extension of the previous approach is the approximation method used in computing the forces of metabolic origin acting on a cell of "arbitrary" shape. The method was sketched in the appendix to the first edition and is more fully developed in the new book. The previous line of attack was through the diffusion equation, a partial differential equation which could be solved explicitly only in very special cases involving highly symmetrical boundary conditions. By means of a bold approximation method, Rashevsky was able to reduce the partial differential equations to ordinary ones and to express the forces in terms of parameters amenable to "rough and ready" measurement: the "long" diameter and the "short" diameter of an "oblong" cell. 